\title{
Bianchi Type- II Universe with Anisotropic Dark Energy in Lyra Geometry
}

\author{
R. K. Dubey ${ }^{1}$, Shri Ram², Neelam Yadav ${ }^{3}$, and Vipendra Dwivedi ${ }^{4}$ \\ ${ }^{1}$ Govt Model Science College, Rewa (M.P.) India \\ ${ }^{2}$ BHU Varanasi (U.P.) \\ ${ }^{3}$ Govt PG College Magaraha Mirzapur (U.P.) \\ ${ }^{4}$ APS University, Rewa (M.P.) India \\ Email: dubeyrk2009@gmail.com, santosh_gdc@rediff.com, rkdubey2004@yahoo.co.in
}

\begin{abstract}
We have investigated a spatially homogeneous and anisotropic Bianchi type II universe filled with anisotropic dark energy within the framework of Lyra geometry with time-varying displacement field vector. The exact solutions of field equations are obtained by using Berman's law of variation for the Hubble parameter that yields a negative constant deceleration parameter. Some physical and kinematical behaviors of the cosmological model are discussed. This model may play relevant and significant roles to study spatially homogeneous cosmologies with anisotropic dark energy and astrophysics.
\end{abstract}

Keywords: Bianchi type II metric, anisotropic dark energy, EoS parameter, constant deceleration parameter.

\section{Introduction}

In the study of modern cosmology immense interest has been shown to study cosmological models with dark energy (DE) in general relativity because of the fact that our observable universe is undergoing a phase of accelerated expansion which has been confirmed by several cosmological observations such as type Ia supernova by several cosmologists [1, 2, 3], Perlmutter et al. [4, 5, 6, 7] etc. Caldwell [8] and Huange [9] have discussed comic microwave background (CMB) anisotropy. Daniel et al. [10] have discussed large scale structure which indicates that the DE dominates the present universe causing cosmic acceleration. The thermodynamical studies of DE reveal that the constituents of DE may be massless particles (bosons and fermions) whose collective behavior resembles with a kind of radiation fluid having negative pressure acting as antigravity responsible for gearing up the universe.

A DE has been considered a kind of fluid satisfying the equation of state $($ EoS $) p=\omega \rho$, where $\rho$ is the density, $\mathrm{p}$ the pressure and $\omega$ may be a time-dependent parameter. Cosmologists have proposed so for various candidates for DE to fit the current observations such as cosmological constant $(\omega=-1)$ phantom $(\omega<-1)$, quintessence fields $(-1<\omega<0)$, etc. Several authors have investigated different aspects of DE models in general relativity with variable EoS parameter. Bamba et al. [11] have reviewed different DE isotropic cosmologies with early deceleration and late-time acceleration. They have studied $\mathrm{f}(\mathrm{R})$ gravity, $\mathrm{f}(\mathrm{R}, \mathrm{T})$ gravity, scalar field theory, holographic DE, coupled DE and cosmological models representing the accelerating expansion with quintessence/phantom nature in details with cosmography tests.

The spatially homogeneous and isotropic models are considered to be most suitable to study large scale structure of the universe. However, it is believed that the early universe may not have been exactly isotropic. This prediction motivates cosmologists to describe early stages of evolution of the universe with models having anisotropic background. Several aspects of anisotropic DE cosmology have been extensively studied by many cosmologists. The EoS parameter of DE can be generalized by determining such a parameter separately in each spatial axis in a consistent way with the considered metric. Rodrigues [12] has investigated a Bianchi type $-\mathrm{I} \wedge \mathrm{CDM}$ model with a non-dynamical component of DE. Koivisto and Mota [13] have presented a cosmological model where the accelerated expansion of the universe is driven by a fluid with an anisotropic EoS parameter by introducing skewness parameters to quantify the deviation of pressure from isotropy. Akarsu and Kilinc [14, 15] have studied Bianchi type -I 
and III cosmological models in the presence of DE and perfect fluid. They considered a phenomenological parameterization of minimally interacting DE in terms to its EoS parameter and timedependent skewness parameters. Kumar and Singh [16] have investigated a spatially homogeneous and anisotropic Bianchi type -1 model with perfect fluid composed of DE and standard visible matter. Pradhan et al. [17] have studied a class of DE models of Bianchi type I with variable EoS parameter and constant deceleration parameter. Adhav et al. [18] have presented a general description of an anistropic DE component by a phenomenological parametrization in terms of EoS parameter and two skewness parameters and obtained the general form of the anisotropic parameter of the expansion for KantowskiSach space-time. Sharif and Zubair [19] have discussed the dynamics of the Bianchi Type I model in the presence of an anisotropic DE and a uniform magnetic. Shairf and Zubair [20] have also investigated Bianchi type-VI cosmological models in the presence of an electromagnetic field and anisotropic DE with constant deceleration parameter. Samanta [21] has investigated Bianchi type III cosmological models with anisotropic DE within the framework of Lyra geometry with the assumptions on the anisotropy of the fluid, power-law and exponential volumetric expansion law. Singh and Sharma [22] considered the totally anisotropic Bianchi type-II space-time metric and have discussed a model of the universe in the presence of an anisotropic DE within the framework of Lyra's manifold with uniform and time-dependent field vector. Shri Ram et al. [23] have investigated a hyper- surfaces homogeneous cosmological model with dynamical equation of state of in Lyra geometry. Recently Shri Ram et al. [24] have presented a Kantowski-Sachs universe filled with an anisotropic DE in Lyra geometry with timedependent displacement vector field. It is worthwhile to mention that Katore et al. [25] have derived a hypersurface-homogeneous cosmological model in the presence of an anisotropic DE in Brans-Dicks gravity theory. Tripathy et al. [26] have studied an LRS Bianchi Type-I model of the universe filled with an anisotropic DE in the framework of the generalized Brans-Dicks gravity theory.

Motivated by the above discussions, we investigate a Bianchi type II universe filled with an anisotropic dark energy in Lyra geometry. The paper is organized as follows. In Sect. 2 the metric and the field equations are presented in Sect. 3, we obtain the exact solutions of the field equations by assuming that the model exhibits constant negative deceleration parameter which is an essential feature of an accelerating universe. We discuss the physical features of the cosmological model in Sect 4, some concluding remarks are given in Sect 5 .

\section{Metric and Field Equations}

We consider the totally anisotropic Bianchi type II space-time in the form

$$
d s^{2}=d t^{2}-A^{2}\left(d x^{2}-z d y^{2}\right)-B^{2} d y^{2}-C^{2} d z^{2}
$$

where $\mathrm{A}(\mathrm{t}), \mathrm{B}(\mathrm{t})$ and $\mathrm{C}(\mathrm{t})$ are comic scale factors.

The energy momentum for anisotropic dark energy in given by,

$$
\begin{aligned}
T_{i}^{j} & =\operatorname{diag}\left[\rho,-p_{x},-p_{y}, p_{z}\right] \\
& =\operatorname{diag}\left[1,-\omega_{x},-\omega_{y},-\omega_{z}\right] \rho
\end{aligned}
$$

where $\rho$ is the energy density of the fluid; $p_{x}, p_{y}$ and $p_{z}$ are pressures on the $\mathrm{x}, \mathrm{y}$ and z-axes respectively. Here $\omega$ is the EoS parameter of the fluid with no deviation and $\omega_{x}, \omega_{y}$ and $\omega_{z}$ are the EoS parameters in the direction of $\mathrm{x}, \mathrm{y}$ and $\mathrm{z}$ axes respectively. We parametrize the energy momentum tensor of the anisotropic DE as

$$
T_{i}^{j}=\operatorname{diag}[1,-(\omega+\delta),-\omega,-\omega] \rho
$$

where $\omega_{y}=\omega_{z}=\omega$ and the skewness parameter $\delta$ is deviation from $\omega$ on the x-axis.

The field equations in Lyra's manifold are [27, 28]

$$
R_{i j}-\frac{1}{2} R g_{i j}+\frac{3}{2}\left(\varphi_{i} \varphi_{j}-\frac{1}{2} g_{i j} \varphi_{\alpha} \varphi^{\alpha}\right)=-T_{i j}
$$

with $8 \pi G=C=1$. Here $\varphi^{i}=(o, o, o \beta(t))$ is the time-like displacement vector field and $\beta(t)$ is the time dependent gauge function. Other symbols have their usual meanings. 
In comoving coordinate system, the field equations (4) for the metric (1) together with (3) yield the following system of equations:

$$
\begin{gathered}
\frac{\ddot{B}}{B}+\frac{\ddot{C}}{C}+\frac{\dot{B} \dot{C}}{B C}-\frac{3}{4} \frac{A^{2}}{B^{2} C^{2}}=-\frac{3}{4} \beta^{2}-(\omega+\delta) \rho \\
\frac{\ddot{C}}{C}+\frac{\ddot{A}}{A}+\frac{\dot{C} \dot{A}}{C A}+\frac{1}{4} \frac{A^{2}}{B^{2} C^{2}}=-\frac{3}{4} \beta^{2}-\omega \rho \\
\frac{\ddot{A}}{A}+\frac{\ddot{B}}{B}+\frac{\dot{A} \dot{B}}{A B}+\frac{1}{4} \frac{A^{2}}{B^{2} C^{2}}=-\frac{3}{4} \beta^{2}-\omega \rho \\
\frac{\dot{A} \dot{B}}{A B}+\frac{\dot{B} \dot{C}}{B C}+\frac{\dot{C} \dot{A}}{C A}-\frac{1}{4} \frac{A^{2}}{B^{2} C^{2}}=\frac{3}{4} \beta^{2}+\rho
\end{gathered}
$$

The conservation equation for the anisotropic fluid can be decomposed into two parts:

$$
\begin{gathered}
\dot{\rho}+(1+\omega) \rho\left(\frac{\dot{A}}{A}+\frac{\dot{B}}{B}+\frac{\dot{C}}{C}\right)+\rho\left(\delta \frac{\dot{C}}{C}\right)=0 \\
\dot{\beta}+\beta\left(\frac{\dot{A}}{A}+\frac{\dot{B}}{B}+\frac{\dot{C}}{C}\right)=0
\end{gathered}
$$

where an over dot denotes derivative with respect to comic time t.

We now define some physical parameters for the metric (1) which are important in cosmological observations. The average scale factor $(a)$ and the volume scalar $(\mathrm{V})$ are defined as

$$
V=a^{3}=A B C
$$

The expansion scalar $(\theta)$, shear scalar $\sigma$, Hubble parameter $(\mathrm{H})$ and the anisotropy parameter $\left(A_{m}\right)$ are given by

$$
\begin{gathered}
\theta=\frac{\dot{A}}{A}+\frac{\dot{B}}{B}+\frac{\dot{C}}{C} \\
\sigma^{2}=\frac{1}{2}\left(\sum_{i=1}^{3} H_{i}^{2}-3 H^{2}\right) \\
H=\frac{1}{3}\left(H_{1}+H_{2}+H_{3}\right) \\
A_{m}=\frac{1}{3} \sum_{i=1}^{3}\left(\frac{H_{i}-H}{H}\right)^{2}
\end{gathered}
$$

where $H_{2}=\frac{\dot{B}}{B}, H_{3}=\frac{\dot{C}}{C}$ are the directional Hubble parameters in the directions of $\mathrm{x}, \mathrm{y}$ and z-axes respectively. $H_{1}=\frac{\dot{A}}{A}$,

The deceleration parameter $q$ in cosmology is the measure of comic acceleration of the universe expansion and is defined as

$$
q=-\frac{a \ddot{a}}{\dot{a}^{2}}
$$

The behavior of the universe models depends on the sign of q. The positive value of q corresponds to a decelerating model while the negative value provides acceleration. It deserves to mention that $q$ was supposed to be positive initially but recent observations from supernova experiments suggest that it is negative.

\section{Solution of Field Equations and Anisotropic DE Model}

We now obtain exact solutions of the field equations (5)-(8) by subtracting (6) from equation (7), 


$$
\frac{\ddot{B}}{B}-\frac{\ddot{C}}{C}+\frac{\dot{A}}{A}\left(\frac{\dot{B}}{B}-\frac{\dot{C}}{C}\right)=0
$$

Equation (17) can be written in the from

$$
\frac{d}{d t}\left(\frac{\dot{B}}{B}-\frac{\dot{C}}{C}\right)+\left(\frac{\dot{A}}{A}+\frac{\dot{B}}{B}+\frac{\dot{C}}{C}\right)\left(\frac{\dot{B}}{B}-\frac{\dot{C}}{C}\right)=0
$$

Integration of (18) yields

$$
\frac{\dot{B}}{B}-\frac{\dot{C}}{C}=\frac{k}{V}
$$

where $\mathrm{k}$ is an arbitrary constant.

To treat equation (19), we can assume the following ad-hoc relationship

$$
A^{m}=B C, m \neq 0
$$

We further assume that $m \neq 0$

$$
B=A^{\frac{m}{2}} D, C=A^{\frac{m}{2}} D^{-1}
$$

where $\mathrm{D}(\mathrm{t})$ is a function of time t. Substituting (21) in equation (19), we obtain

$$
\frac{\dot{D}}{D}=\frac{K}{A^{m+1}}
$$

where $\mathrm{K}$ is an arbitrary constant of integration. From equation (11) and (20), we get

$$
V=A^{m+1}=a^{3}
$$

Then equation (22) becomes

$$
\frac{\dot{D}}{D}=\frac{K}{a^{3}}
$$

We now obtain a cosmological model with negative constant deceleration parameter. Berman [29] proposed a law of variation for Hubble parameter that yields a constant value of deceleration parameter. For an accelerating model of the universe, we take the constant negative. Then equation (14), on integration, gives

$$
a=\left(c_{1} t+c_{2}\right)^{\frac{1}{1+q}}
$$

where $c_{1}$ and $c_{2}$ are integration constants. This equation implies that the condition of expansion is $1+q>0$. Substituting (25) in equation (24) and integrating the result, we obtain

$$
D=K_{1} \exp \left[\frac{K(1+q)}{c_{1}(q-2)}\left(c_{1} t+c_{2}\right)^{\frac{q 2}{q+1}}\right]
$$

where in constant of integration. We take $K_{1}=1$ without loss of generality. Therefore the explicit solutions of the scale factors $\mathrm{A}, \mathrm{B}$ and $\mathrm{C}$ are

$$
\begin{gathered}
A=\left(c_{1} t+c_{2}\right)^{\frac{3}{(m+1)(1+q)}} \\
B=\left(c_{1} t+c_{2}\right)^{\frac{3 m}{2(1+q)(m+1)}} \exp \left[\frac{K(1+q)}{C_{1}(q-2)}\left(C_{1} t+C_{2}\right)^{\frac{q-2}{1+q}}\right] \\
K_{1} C=\left(c_{1} t+c_{2}\right)^{\frac{3 m}{2(1+q)(m+1)}} \exp \left[\frac{-K(1+q)}{c_{1}(q-2)}\left(c_{1} t+c_{2}\right)^{\frac{q-2}{1+q}}\right]
\end{gathered}
$$

From equations (10) (11) and (25), we obtain the solution for the gauge function in the form

$$
\beta=\frac{\beta_{o}}{\left(c_{1} t+c_{2}\right)^{\frac{3}{1+q}}}
$$

where $\beta_{o}$ is an arbitrary constant. 
Making use of equations (27), (28), (29) and (30) in equations (5)-(8), we obtain the energy density, EoS parameter and skeness parameter as

$$
\begin{gathered}
\rho=\frac{9 m(m+4) c_{1}^{2}}{4(1+q)^{2}(m+1)^{2}\left(c t+c_{2}\right)^{2}}+\frac{3 \beta_{o}^{2}-4 K^{2}}{4\left(c_{1} t+c_{2}\right)^{\frac{6}{1+q}}}-\frac{1}{4\left(c_{1} t+c_{2}\right)^{\frac{6(m-1)}{(m+1)(1+q)}}} \\
\omega=-\frac{1}{\rho}\left[\frac{9\left(m^{2}+2 m+4\right) c_{1}^{2}}{4(1+q)^{2}(m+1)^{2}\left(c_{1} t+c_{2}\right)^{2}}+\frac{4 K^{2}-3 \beta_{o}^{2}}{\left(c_{1} t+c_{2}\right)^{\frac{6}{1+q}}}-\frac{3 c_{1}^{2}(m+2)}{2(m+1)(1+q)\left(c_{1} t+c_{2}\right)^{2}}+\frac{1}{4\left(c_{1} t+c_{2}\right)^{\frac{6(m-1)}{(m+1)(1+q)}}}\right] \\
\delta=\frac{1}{\rho}\left[\frac{3 c_{1}^{2}(m-2)}{2(1+q)(m+1)\left(c_{1} t+c_{2}\right)^{2}}-\frac{1}{4\left((1+q)^{2}(m+1)^{2}\left(c_{1} t+c_{2}\right)^{2}\right)}+\frac{9 c_{1}^{2}\left(m^{2}-4\right)}{\left(c_{1} t+c_{2}\right)^{\frac{6(m-1)(m+1)(1+q)}{(m-1)}}}\right]
\end{gathered}
$$

Hence, the metric (1) together with equations (27)-(33) constitutes a Bianchi type-II cosmological model in the presence of an anisotropic DE in Lyra geometry.

The directional and mean Hubble parameters have the values:

$$
\begin{gathered}
H_{1}=\frac{3 c_{1}}{(m+1)(1+q)\left(c_{1} t+c_{2}\right)} \\
H_{2}=\frac{3 m c_{1}}{2(m+1)(1+q)\left(c_{1} t+c_{2}\right)}+\frac{K}{\left(c_{1} t+c_{2}\right)^{\frac{3}{1+q}}} \\
H_{3}=\frac{3 m c_{1}}{2(m+1)(1+q)\left(c_{1} t+c_{2}\right)}-\frac{K}{\left(c_{1} t+c_{2}\right)^{\frac{3}{1+q}}} \\
H=\frac{c_{1}}{(1+q)\left(c_{1} t+c_{2}\right)}
\end{gathered}
$$

The expansion $\theta$ and $\sigma$ have the values given by

$$
\begin{gathered}
\theta=\frac{3 c_{1}}{(1+q)\left(c_{1} t+c_{2}\right)} \\
\sigma^{2}=\frac{3 c_{1}^{2}(m-2)^{2}}{(m+1)^{2}(1+q)^{2}\left(c_{1} t+c_{2}\right)^{2}}+\frac{K^{2}}{\left(c_{1} t+c_{2}\right)^{\frac{6}{1+q}}}
\end{gathered}
$$

The mean anisotropy parameter $A_{m}$ becomes

$$
A_{m}=\frac{(m-2)^{2}}{2(m+1)^{2}}+\frac{2 K c_{1}^{2}(1+q)^{2}}{3\left(c_{1} t+c_{2}\right)^{\frac{4-2 q}{1+q}}}
$$

\section{Some Physical Features of the Model}

We now discuss physical and geometrical behaviors of the derived model. For the present model we observe that the spatial volume is zero at the time $t=-\frac{c_{2}}{c_{1}}$. At this epoch the physical parameters are infinite. Therefore the universe exhibits a point-type singularly at $t=-\frac{c_{2}}{c_{1}}$. The initial time of the universe can be shifted to zero by taking $c_{1}=1$ and $c_{2}=0$. 
Since we study the universe filled with DE fluid, we are interested in studying relatively late-time universe rather than the early universe. The model is well behaved in the range $-\frac{c_{2}}{c_{1}}<t<\infty$. At the initial singularity the physical parameters $\theta$ and $\sigma$ tend to infinite. So the universe starts evolving from initial singularity with infinite rate of expansion and shear which ultimately tend to zero as $t \rightarrow \infty$. For large time the spatial volume becomes infinitely large and the expansion stops.

From equations (40), we see that the anisotropic parameter of expansion $A_{m}$ is infinite at the initial time which shows that the model is highly anisotropic at the time of evolution of the universe. The parameter $A_{m}$ tends to a constant as $t \rightarrow \infty$, showing that the universe is anisotropic for all times.

- The energy density of the dark fluid is infinite at $t=0$. Therefore the model evolves with infinite density and ultimately becomes an empty space for large time if $m>1$. Thus we have a constraint over $\mathrm{m}$.

- The deviation free EoS parameter $\omega$, being infinite at the initial time, is a decreasing function of time and ultimately tends to a negative constant value

$$
\omega=-\frac{m^{2}+2 m+4}{m(m+4)}
$$

For large time $\omega=-1$ if $\mathrm{m}=2$. Therefore the model represents a vacuum universe and mathematically equivalent to cosmological constant as the source of DE. Similarly it is observed that as $t \rightarrow \infty, \omega<-1$ (techyon region) and $\omega>-1$ (quintessence region) according as $\mathrm{m}>2$ and $\mathrm{m}<2$. The skewness parameter $\delta$ is infinite at $\mathrm{t}=0$ and tends to zero as $t \rightarrow \infty$.

- In the present model the gauge function $\beta$ is infinite at the initial singularity. From (30), we see that $\beta$ tends to zero as $t \rightarrow \infty$. Therefore the concept of Lyra manifold is meaningful for finite time, but does not remain for very large time.

\section{Conclusion and Perspectives}

In this paper, we have examined the behaviors of a spatially homogeneous and totally anisotropic Bianchi type II cosmological model in the presence of an anisotropic DE with negative constant deceleration parameter within the framework of lyra's manifold with time-varying displacement field vector which is capable for describing certain attributes of the universe for suitable values of certain parameters. The derived model of the universe has singularity at time $t=-\frac{c_{2}}{c_{1}}$. The universe starts evolving at the initial singularity with infinite rate of expansion which vanishes for large time. The anisotropy of the expansion converges to a constant as $t \rightarrow \infty$ which shows that the universe is anisotropic for all times. The EoS parameter $\omega$ is time-varying and decreasing function of time which attains a negative constant value for large time.

It is seen that for later or times $\omega$ may be in the phantom region $(\omega<-1)$ or in region dominated by cosmological constant $(\omega=-1)$ or in the quintessence region $(\omega>-1)$ according as $\mathrm{m}>2$ or $\mathrm{m}=2$ or $\mathrm{m}<2$. These results demonstrate convincingly anisotropic expansion of the universe which is consistent with recent observational data.

\section{References}

1. A.G. Reiss, et al. "Observable Evidence from Supernovac for an Accelerating Universe a Cosmological Constant," Astron J. 116, 1009-1038 (1998).

2. A.G. Reiss, et al, "The Case for an Accelerating Universe from Supernovac," Publ; Astron, Soc, Pac. $1141284-$ 1299 (2000).

3. A.G. Reiss, et al., "Type -Ia Supernova Discoveries at $1|z|>1$ from the Hubble Telescope, Evidence for Past Deceleration and Constraints and Dark Energy Evolution," Astrophys J.607, 665-687 (2004).

4. S. Perlmutter, et al. "Measurement of the Cosmological Parameters $\Omega$ and $\wedge$ from the First Seven Supernovae at $z \geq 0.35$," Astrophys J. 483, 565-581 (1997). 
5. S. Perlmutter, et al, "Discovery of a Supernovac Explosion at Half the Age of the Universe," Nature, 391, 51-54 (1998).

6. S Perlmutter, et al, "Measurements of Omega and Lambda from 42 High-Red Shift Supernovac," Astrophys, J. 517, 565-586 (1999).

7. R.K. Knop, et al, (Supernova Cosmology Project collaboration) "New Constraints on $\Omega \mathrm{m} \Omega \Lambda$ and $\omega$ from an Independent Set of 11 High Red- Shift Supernovac Observed with the Hubble Space Telescope," Astrophys. J. 598, 102-137 (2003).

8. R.R. Caldwell, "A Phantom Menace Cosmological Consequences of a Dark Energy Component with SuperNegative Equation of State," Phys. Lett. B 545, 23-29 (2002).

9. Huange, et al., "Holographic Explanation of Wide Angle Power Correlation suppresion in the comic microwave background radiation," J. Cosmol Astropart Phys. 05, 013 (2006).

10. Daniel, et al., Large "Scale Structure as a Probe of Gravitational Slip," Phys. Rev. D77, 103513 (2008).

11. K. Bamba, et al., "Dark Energy Cosmology: The Equivalent Description Via Different Theoretical Models and Cosmography Tests," Astrophys Space Sci. 342, 155-228 (2012).

12. D.C. Rodrigues, "Anisotropic Cosmological Constant and the CMB Quadruple Anomally," Phys Rev. D77, 023534-023542 (2008).

13. T. Koivisto and D.F. Mota, "Accelerating Cosmologies with an Anisotropic Equation of State", Astrophys J. $679,1-5(2008)$.

14. O. Akarsu, C.B. Kilinc, "de sitter Expansion with Anisotropic Fluid in Bianchi Type- I Space Time", 326, 315$322(2010)$.

15. O. Akarsu and C.B. Kilinc, "Bianchi Type- III models with Anisotropic Dark Energy," Gen. Relativ. Gravit. 42. 763-775 (2010).

16. S. Kumar and C.P. Singh, "Anisotropic Dark Energy Model with constant Deceleration Parameter," Gen. Relativ. Gravit. 43, 1427-1442 (2011).

17. A. Pradhan, et al., "Bianchi Type-I Anisotropic Dark Energy Model," Int. J. Theor. Phys. 50, 2923-2938 (2011).

18. K.S. Adhav, et al. "Kantowski- Sachs Cosmological Models with Anisotropic Dark Energy," Cent. Eur. J. Phys. 9, 919-925 (2011)

19. M. Sharif, M. Zubair, "Dynamics of Bianchi Type- I Universe with Magnetized Dark Energy"' Astrophys Space Sci. 330, 339-346 (2010)

20. M. Sharif and M. Zubair, "Dynamics of a Magnetized Bianchi Type-VI," Universe with Anisotropic Fluid, Astrophys. Space, Sci. 339, 45-51 (2013).

21. G.C. Samanta, "Bianchi Type - III Cosmological Models with Anisotropic Dark Energy," Int. J. Theor. Phys. $52,3442-3456$ (2013).

22. J.K. Singh and N.S. Sharma, "Spatially, Homogenous Bianchi Type- II Universe with Anisotropic Dark Energy in Lyra's Geometry," The African Review of Physics. 8, 0056 397-402 (2013).

23. Shri Ram, et al., "Hypersurface- Homogeneous Cosmological Models with Dynamical Equation of State Parameter in Lyra's Geometry," Canadian J. Phys. 93, 1009-104 (2015)

24. Shri Ram, et al., "Kantowski-Sachs universe with Anisotropic Dark Energy in Lyra Geometry," Chinese J Phys 54, 953-960 (2016)

25. S.O. Katore, et al, "Hypersurface - Homogeneous Space - Time Anisotropic Dark Energy in Brans-Dicke Theory of Gravitation," Commun. Theor. Phys. 62, 768 - 774 (2014).

26. Sunil K. Tripathy, et al," Unified Dark Fluid in Brans-Dicke Theory," Eur, Phys. J. C. 75, 148 -157 (2015).

27. D.K. Sen, "A Static Cosmological Model," Z. Phys. 149, 311-323 (1975).

28. D. K. Sen and K. A. Dunn, "A Scalar-Tensor Theory of Gravitation in a Modified Riemannian Manifold," J. Math. Phys. 12, 578 (1971)

29. M.S. Berman, "Special Law of Variation for Hubble Parameter," Nuovo Cimento B 74, 182 - 186 (1983). 